\title{
Determinación de Actitudes Posturales tras el Abordaje Ortopédico Funcional. Estudio Descriptivo
}

\author{
Determination of Postural Attitudes and their Evolution after \\ Functional Orthopedic Approach. Descriptive Study
}

\author{
María Berenice Enríquez Núñez; Cristal Yurixie Díaz Rosas; Martha Elena Hernández Montoya; \\ Jesús Alberto Luengo Fereira \& Heraclio Reyes Rivas
}

ENRÍQUEZ, M. B.; DÍAZ, C. Y.; HERNÁNDEZ, M. E.; LUENGO, J. A. \& REYES, H. Determinación de actitudes posturales tras el abordaje ortopédico funcional. Estudio descriptivo. Int. J. Odontostomat., 12(1):113-119, 2018.

RESUMEN: El objetivo del estudio fue determinar las actitudes posturales tras el uso de aparatos Ortopédicos Funcionales (AOF) en pacientes pediátricos con maloclusión. Estudio descriptivo, exploratorio, longitudinal, realizado en las instalaciones de la Clínica de la Especialidad en Odontopediatría (CLIO) de la UAO/UAZ, durante el periodo de Enero a Septiembre de 2015. Se seleccionaron pacientes de ambos sexos con edades entre 6 y 12 años, a través de un muestreo no probabilístico a conveniencia. Como variables se determinó la presencia o ausencia de equilibrio postural a través de fotografías en vistas anterior, posterior y lateral (derecha/izquierda) en tres momentos: previo a la instalación del AOF, 10 minutos después y posterior a 10 semanas de uso. Se consideró para la evaluación, el tipo de maloclusión de acuerdo a Angle, el AOF utilizado, y la cantidad de parámetros en desequilibrio en E0 y E2. Los datos recolectados fueron almacenados en el programa SPSS V-21 y analizados con las pruebas chi cuadrada y Wilcoxon, considerando significativo un valor de $p<0,05$. Fueron evaluados 10 pacientes con una edad media de 7,2 $\pm 0,99$ años que presentaron en su totalidad actitudes posturales anómalas en los planos sagital y frontal. Fue posible determinar una reducción de 60 y $80 \%$ de desequilibrio en algunos de los parámetros evaluados (simetría de hombros y la altura en punta de los dedos), y estos cambios se manifestaron desde forma sutil desde la instalación del AOF y se incrementaron en la E2. La terapia miofuncional sobre pacientes con maloclusiones I y II de Angle se acompaña de cambios en la actitud postural que pueden ser observables desde la instalación de los dispositivos, y éstos cambios permanecen durante el periodo de uso.

PALABRAS CLAVE: actitud postural, maloclusión, aparatos ortopédicos funcionales.

\section{INTRODUCCIÓN}

El cuerpo humano se mantiene de pie gracias al equilibrio que existe entre todas las estructuras que le componen, una alteración a cualquier nivel repercute en mayor o menor medida sobre sus demás componentes (Silvestrini-Biavati et al., 2013). Las modificaciones en la posición y/o forma de maxilares o dientes, trae consigo un cambio en la posición de la columna vertebral, piernas, pies, cadera, y otras estructuras, y de igual manera una alteración en los miembros inferiores puede ocasionar modificaciones en la posición de maxilares, al grado de provocar una respiración o deglución incorrectos (Martínez Medina et al., 2003).
La relación biomecánica entre la cabeza, la columna cervical y órganos dentarios han generado un amplio debate científico, por la estrecha interrelación que mantienen como conjunto estructural y se ha logrado determinar la existencia de problemas posturales en más de $90 \%$ de los casos de sujetos con algún tipo de maloclusión (Pereira Rosa et al., 2008; Baldini et al., 2013; Murrieta Pruneda, 2013; Aguilar Moreno \& Taboada Aranza, 2013).

Actitud postural. La Academia Americana de Ortopedia (AAO) considera la actitud postural como el equilibrio entre los músculos y huesos que protege a las 
ENRÍQUEZ, M. B.; DÍAZ, C. Y.; HERNÁNDEZ, M. E.; LUENGO, J. A. \& REYES, H. Determinación de actitudes posturales tras el abordaje ortopédico funcional. Estudio descriptivo. Int. J. Odontostomat., 12(1):113-119, 2018.

demás estructuras del cuerpo contra el trauma, de forma situación dinámica y de adaptación constante a los estímulos recibidos, y que refleja experiencias momentáneas. Para que esto ocurra es imprescindible un tono muscular adecuado y flexibilidad (Garcia de Paula e Silva et al., 2008; Montero Parrilla \& Denis Alfonso, 2013).

El cuerpo requiere una actitud postural estable y balanceada de sostén, en la cual los movimientos voluntarios y coordinados puedan realizarse de forma orgánica como parte de las funciones naturales (Garcia de Paula e Silva et al.; Montero Parrilla \& Denis Alfonso), y con el menor consumo energético posible (Novo et al., 2013), al ejercer una función muscular necesariamente otro grupo debe promover la estabilidad y posicionamiento de las estructuras óseas (Garcia de Paula e Silva et al.). La ruptura del equilibrio ocasionada por alteraciones propias de los miembros pueden dar origen a un grupo de procesos patológicos (Novo et al.). El ser humano busca continuamente el equilibrio corporal, y posee la capacidad de buscarlo cuando lo ha perdido, esto en el ámbito clínico se denomina estabilidad o control postural (Montero ParriIla \& Denis Alfonso).

Alteraciones en la actitud postural. La mala actitud postural, comprende un proceso de lesiones motoras repetitivas por una deficiente biomecánica que crea palancas ocasionando múltiples sobrecargas de trabajo para los sistemas articulares (Montero Parrilla \& Denis Alfonso). Una gran mayoría de éstos problemas tiene lugar durante la infancia por la adopción de actitudes incorrectas, ocasionando defectos estéticos, desórdenes cráneo-faciales y en la actividad funcional durante la respiración, deglución, circulación y locomoción (Aguilar Moreno \& Taboada Aranza).

Síndromes disfuncionales. La correlación entre el componente estomatognático y el resto del cuerpo se origina a través del sistema neuromuscular, por medio de cadenas musculares a lo largo del cuerpo. Son cinco las cadenas descritas por Struyf-Denys (1978); a) la cadena anterior (hioideos, orbicular de los labios, pterigoideos externos y lengua), b) anterior lateral (maseteros, temporales, pterigoideos internos y esternocleidomastoideo), c) posterior (espinales cervicales), d) posterior lateral (temporales y trapecios), y e) central (lengua). Los músculos masticadores pertenecen a más de una cadena, creando así un vínculo regional, y a su vez con el resto del cuerpo. Entre los factores que condicionan de manera determinante el funcionamiento normal o anormal del sistema de equi- librio mecánico, se encuentra la oclusión, que interviene de manera determinante en el complejo muscular de flexión de la cabeza en relación con el tronco (Novo et al.).

Síndrome postural descendente (SPD). Se origina por maloclusiones dentarias o lesiones de la articulación temporomandibular (ATM) y repercute en la actitud postural del individuo, quien en búsqueda de una línea bipupilar paralela que lo haga sentirse más estable empieza a adoptar una serie de contracciones musculares inadecuadas que generan una postura incorrecta provocando deformidad en la columna vertebral y piernas (Machado et al., 2009; Cavallé et al., 2012). Cuando se producen cambios por sobrecargas bien sea por lesiones en ATM o por maloclusiones, las contracciones musculares a nivel del sistema estomatognático influyen sobre la posición del maxilar inferior. La aparición de sobrecargas crea una curvatura en la columna vertebral, cambiando el equilibrio de la cabeza sobre la columna, el maxilar inferior tiende a lateralizarse hacia el lado opuesto de la inclinación de la cabeza. En un paciente se percibe un hombro más bajo, la cabeza se inclina hacia el lado opuesto y el maxilar inferior se lateraliza hacia el lado en que se observa el hombro más bajo; se origina una función asimétrica del maxilar inferior, la oclusión presenta líneas medias que no coinciden y el plano oclusal puede estar inclinado en su vista frontal (Machado et al.; Murrieta Pruneda).

Síndrome postural ascendente (SPA). En éste, el desequilibrio del aparato locomotor produce variaciones que pueden influir en el sistema estomatognático. Pueden estar involucrados los músculos masticadores, la articulación temporomandibular y/o la oclusión. Ejemplo de ello son las personas con pie plano, el cual apoya completamente la planta del pie sobre el suelo debido a la ausencia del arco medio. En este caso el hundimiento plantar propicia que busque una mejor postura compensatoria y como consecuencia una protrusión mandibular (Machado et al.; Murrieta Pruneda).

Síndrome postural mixto (SPM). Se caracteriza por la presencia simultánea de ambos síndromes (ascendente y descendente).

Está poco documentado el mecanismo de influencia de la postura corporal sobre las alteraciones oclusales y viceversa, y en menor medida la terapéutica de abordaje, existen reportes del uso de dispositivos ortodónticos, placas acrílicas, entre otros dispositivos (Miralles et al., 1997; Chessa et al., 2001) y el 
ENRÍQUEZ, M. B.; DÍAZ, C. Y.; HERNÁNDEZ, M. E.; LUENGO, J. A. \& REYES, H. Determinación de actitudes posturales tras el abordaje ortopédico funcional. Estudio descriptivo. Int. J. Odontostomat., 12(1):113-119, 2018.

abordaje quiropráctico por otro lado (Knutson \& Jacob, 1999). El objetivo de este estudio es registrar la actitud postural anterior, posterior y lateral, antes y después del uso de Aparatos Ortopédicos Funcionales (AOF) en pacientes con algún tipo de maloclusión, y su posible asociación.

\section{MATERIAL Y MÉTODO}

Diseño y Población de Estudio. Se trata de un estudio exploratorio, descriptivo y longitudinal. Se consideraron pacientes que asistieron a la clínica de la especialidad en Odontopediatría (CLIO /UAZ) durante el periodo de enero a septiembre del 2015. Se seleccionaron niños de ambos sexos, de 6 a 12 años de edad, cuyo expediente clínico-odontológico estuviera completo y con necesidades de atención por maloclusión con un Aparato Ortopédico Funcional (AOF). Se excluyeron pacientes con antecedentes de lesiones cráneo-cervicales previas y síndromes asociados. Una vez obtenido el consentimiento/asentimiento de los padres y menores se procedió a la realización de un examen clínico bucal y de actitud postural (AP).

Muestra. Se realizó un muestreo no probabilístico por conveniencia sobre la cantidad de individuos que desearan participar y contaran con el informe de consentimiento informado.

Procedimientos. Un odontólogo entrenado se encargó de establecer el tipo de maloclusión de acuerdo a la clasificación propuesta por Angle (1889) mediante un examen bucal completo en un sillón dental y con luz artificial, con las barreras especificadas en la NOM-013-SSA2-2006. Se solicitó al paciente morder en repetidas ocasiones hasta obtener un registro confiable, y se obtuvo la relación molar de ambos lados (Cl, II o III). Los dispositivos utilizados para el tratamiento de la maloclusión fueron el modelador elástico Bimler (MBim), Pistas Planas Indirectas (PPI) o Simoes Network (SN).

Se realizó la captura fotográfica de la actitud postural (AP) en las vistas laterales (izquierda y derecha), anterior y posterior con una cámara Cannon PowerShotÒ SX500 IS, con objetivo de 24 mm y un trípode profesional para fotografía Giottos IY442 a una distancia de 2 a 2,5 $\mathrm{m}$ de acuerdo al procedimiento sugerido por Kendall \& McCreary (1995), colocando al paciente en posición ortostática relajada, descalzo y con ropa cotidiana (Kendall \& Mc Creary;
Garcia de Paula e Silva et al.), ésta maniobra fue realizada por un investigador independiente, previamente entrenado, que no participo en la selección de los casos.

La AP de los participantes fue evaluada antes de la colocación del AOF (E0), 10 min después de su colocación (E1) y la tercera a las 10 semanas de uso del AOF (E2), bajo la supervisión de un fisioterapeuta calificado y sobre la secuencia fotográfica realizada.

Evaluación postural. Se trazaron líneas verticales y horizontales sobre las estructuras de referencia en los registros fotográficos desde cuatro vistas, y a partir de su relación con las estructuras de referencia se determinó el equilibrio postural. En un individuo equilibrado, el hilo con plomada pasa por las siguientes estructuras (Pereira Rosa et al.):

- Vista frontal: entre las articulaciones de la rodilla, a través de la sínfisis púbica, sobre la cicatriz onfálica, sobre el proceso xifoides, sobre la punta de la nariz. En este plano al igual que la vista posterior, se evalúa la posición cefálica, simetría de hombros, nivelación de la pelvis y altura de la punta de los dedos de manos.

- Vista posterior: entre las articulaciones de las rodillas, sobre el pliegue de los glúteos, por los cuerpos vertebrales y el proceso espinoso de la vértebra cervical C7.

- Vista Lateral: ligeramente anterior al eje de la articulación de la rodilla, por el cuerpo de las vértebras lumbares, por el proceso odontoides del axis y por el meato auditivo externo. Se evaluó la posición de la cabeza, curvas fisiológicas del cuerpo: cervical, torácica y lumbar, además de la caída de la plomada en el pie.

Se consideró equilibrada cuando se observaron las relaciones estructurales anteriormente descritos, y desequilibrada al mostrar alguna alteración en los parámetros evaluados (Figs. 1 y 2).

Abordaje con AOF. La confección de los AOF fue realizada por un clínico especialista en ortopedia funcional de los maxilares experimentado. Se recomendó uso de 24 h al día, retirándole únicamente para la alimentación e higiene. Para los pacientes cuyos AOF contenían accesorios de activación en casa, se indicó $0,25 \mathrm{~mm}$ una o dos veces a la semana, de acuerdo a cada caso en particular. 

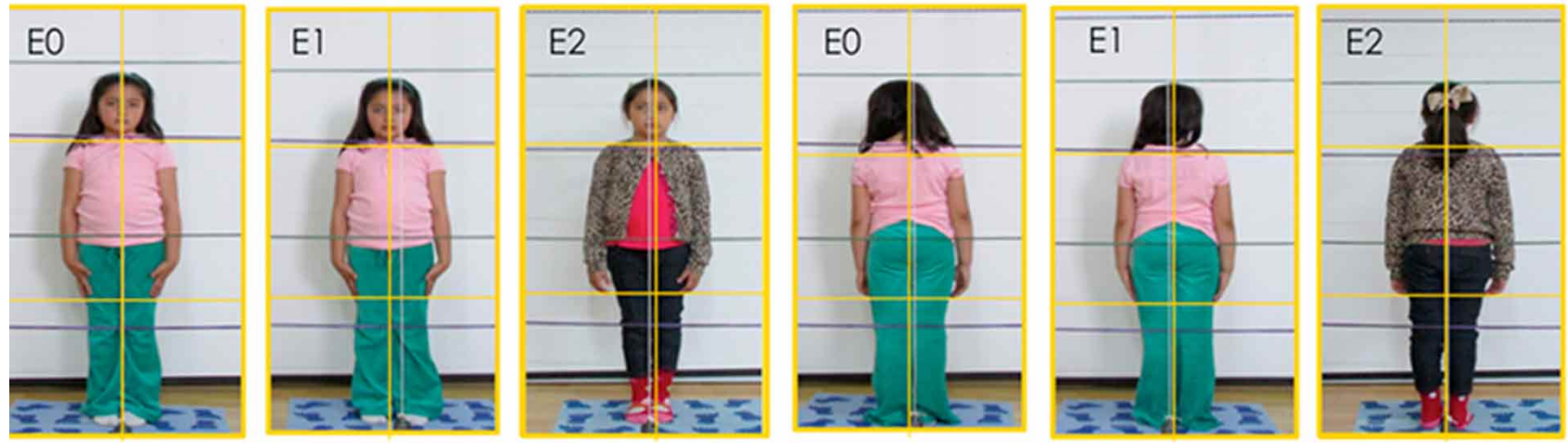

Fig. 1. E0 de pacientes femenino de 6 años de edad. Hilo y plomada ayudan a determinar desequilibrio cefálico con inclinación hacia la izquierda. Además se aprecia una diferencia en la altura de ambos hombros y caída mas baja de los dedos del mismo lado. En E1 y E2 se aprecia diferencias claras sobre la altura de hombros, punta de dedos y posición cefálica.
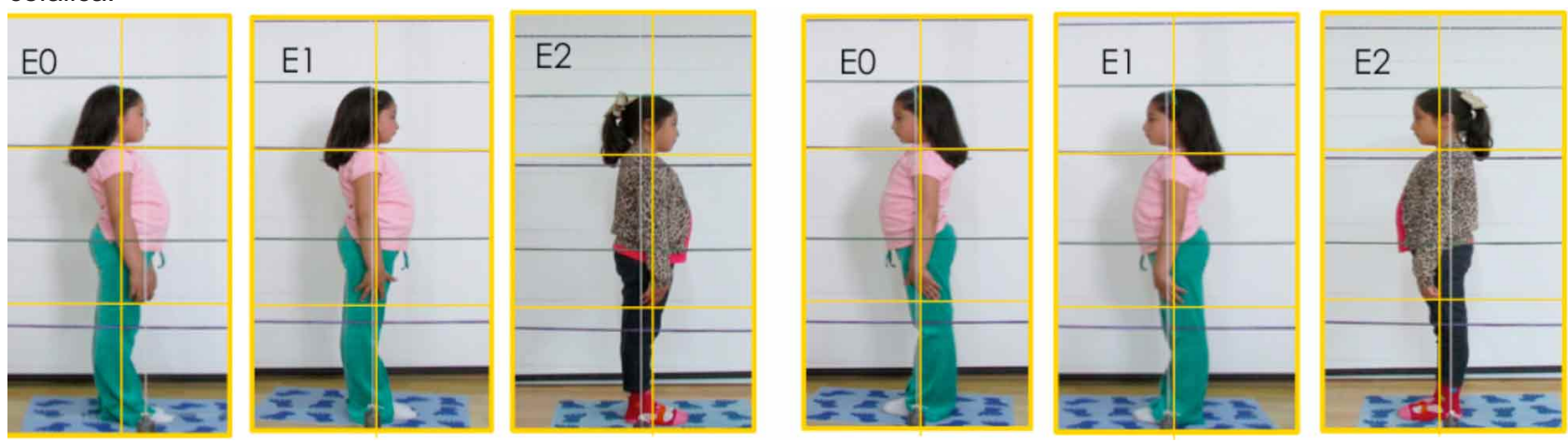

Fig. 2. En las vistas laterales (misma Paciente) se registraron las curvas fisiológicas de la columna, lordosis cervical, cifosis torácica y lordosis lumbar, la caída de la plomada en los pies; y la posición de cabeza se registro como recta o con proyección anterior/posterior. En E0, se aprecia una posición Cefálica y caída recta de Hilo en los pies lordosis cervical normal, flecha intermedia mostrando cifosis torácica normal, flecha inferior mostrando lodosis lumbar aumentada en el mismo paciente. En E1 permanece la lordosis pronunciada y en E2 se observa una reducción de la misma.

Análisis estadístico. El procesamiento de datos y análisis estadísticos se realizó con el programa SPSS v.21. Se analizó la frecuencia de las variables de acuerdo a la clase de Angle, al género, así los parámetros de postura de acuerdo a las variables registradas con una prueba de Chi Cuadrado. Se aplicó una prueba de Wilcoxon para determinar las diferencias entre la cantidad de parámetros AP antes y después del abordaje. Se consideraron diferencias estadísticamente significativas cuando el valor de $p$ fue $<0.05$.

\section{RESULTADOS}

Se logró seleccionar a 10 pacientes que cumplieron con los criterios de inclusión establecidos, cinco de sexo femenino y cinco masculino, con una edad media de 7.2 \pm 0.99 . Se determinó en el examen facial el $90 \%$ de pacientes con perfil convexo. Se observó clase I de Angle en 6 pacientes (60 \%), y Clase II en 4 (40\%). Los AOF utilizados por los pacientes fueron cinco PPI (de clases I y II); además de tres MBim (A, B y C), y finalmente dos dispositivos SN del tipo 2.

Evaluación Postural. En la E0 de las vistas anterior/posterior, los parámetros simetría de hombros y altura de la punta de los dedos fueron los más afectados, la totalidad de los participantes mostraron algún tipo de desequilibrio hacia el lado derecho o izquierdo (Tabla I), seguido de la simetría cefálica en el $20 \%$ y finalmente, la nivelación de la cadera fue el aspecto más equilibrado con $50 \%$.

La evaluación E1, refleja una sutil influencia del AOF sobre la postura, al observarse incremento 
ENRÍQUEZ, M. B.; DÍAZ, C. Y.; HERNÁNDEZ, M. E.; LUENGO, J. A. \& REYES, H. Determinación de actitudes posturales tras el abordaje ortopédico funcional. Estudio descriptivo. Int. J. Odontostomat., 12(1):113-119, 2018.

Tabla I. Evaluación de las actitudes posturales en los 3 momentos (E0, E1 y E2), en las vistas anterior y posterior.

\begin{tabular}{|c|c|c|c|c|c|c|c|c|}
\hline \multirow{2}{*}{$\begin{array}{c}\text { AP } \\
\text { Anterior y } \\
\text { posterior }\end{array}$} & \multicolumn{2}{|c|}{ Simetría cefálica } & \multicolumn{2}{|c|}{ Simetría de hombros } & \multicolumn{2}{|c|}{$\begin{array}{c}\text { Altura de punta de los } \\
\text { dedos }\end{array}$} & \multicolumn{2}{|c|}{ Nivelación de caderas } \\
\hline & $\mathrm{Eq}$ & Deseq. (DI/DD) & $\mathrm{Eq}$ & $\begin{array}{l}\text { Deseq. } \\
\text { (DI/DD) }\end{array}$ & $\mathrm{Eq}$ & $\begin{array}{l}\text { Deseq. } \\
\text { (DI/DD) }\end{array}$ & $\mathrm{Eq}$ & Deseq. (DI/DD) \\
\hline E0 & 2 & $8(4 / 4)$ & 0 & $10(5 / 5)$ & 0 & $10(5 / 5)$ & 5 & $(1 / 4)$ \\
\hline E1 & 5 & $5(2 / 3)$ & 3 & $7(4 / 3)$ & 3 & $7(4 / 3)$ & 7 & $(1 / 2)$ \\
\hline E2 & 6 & $4(2 / 2)$ & 6 & $4(2 / 2)$ & 8 & $2(1 / 1)$ & 10 & $(0 / 0)$ \\
\hline \multirow[t]{2}{*}{$\%$ Equ. } & $40 \%$ & & $60 \%$ & & $80 \%$ & & $50 \%$ & \\
\hline & \multicolumn{2}{|c|}{$p=0,037 ; \quad$ IC $0,03-0,76$} & \multicolumn{2}{|c|}{$p=0,168 ; I C \quad 0,203-1$} & \multicolumn{2}{|c|}{$p=0,000 ; \quad I C \quad 0,49-1,10$} & \multicolumn{2}{|c|}{$p=0,015 ; \quad I C \quad 0,123-0,877$} \\
\hline
\end{tabular}

Tabla II. Evaluación de las actitudes posturales en los 3 momentos (E0, E1 y E2), en las vistas laterales izquierda y derecha.

\begin{tabular}{|c|c|c|c|c|c|c|c|c|c|c|c|c|}
\hline \multirow{2}{*}{$\begin{array}{l}\text { Vis tas } \\
\text { Laterales } \\
\text { Izq/Der }\end{array}$} & \multicolumn{3}{|c|}{ Posición cefálica } & \multicolumn{2}{|c|}{ Lordosis cervical } & \multicolumn{2}{|c|}{ Cifosis torácica } & \multicolumn{2}{|c|}{ Lordosis lumbar } & \multicolumn{3}{|c|}{$\begin{array}{l}\text { Caída del hilo } \\
\text { en los pies }\end{array}$} \\
\hline & $\mathrm{R}$ & PA & PP & Normal & Aumentada & Normal & Aumentada & Normal & Aumentada & $\mathrm{R}$ & $\mathrm{CA}$ & $\mathrm{CP}$ \\
\hline E0 & 5 & 5 & 0 & 4 & 6 & 10 & 0 & 1 & 9 & 5 & 5 & 0 \\
\hline E1 & 7 & 3 & 0 & 5 & 5 & 10 & 0 & 1 & 9 & 5 & 5 & 0 \\
\hline E2 & 10 & 0 & 0 & 9 & 1 & 10 & 0 & 6 & 4 & 6 & 4 & 0 \\
\hline \%Equ. & $\begin{array}{r}50 \% \\
p=0,015 ;\end{array}$ & $123-$ & & $\begin{array}{c}50 \% \\
p=0,015\end{array}$ & $0,123-0,877$ & $-\overline{S i n}$ & erencias & $\begin{array}{c}50 \% \\
p=0,015\end{array}$ & $0,123-0,877$ & $\begin{array}{l}10 \% \\
p=0,3\end{array}$ & IC 0,1 & \\
\hline
\end{tabular}

Recta (R); Posición anterior (PA); Posición posterior (PP); Caída anterior (CA); Caída posterior (CP); Porcentaje de equilibrio alcanzado (\%Equ.)

en el equilibrio en todos los aspectos. En la E2 esta se vuelve más contundente al registrarse un incremento que osciló del $40 \%$ al $80 \%$ de equilibrio los parámetros evaluados, con un mayor porcentaje en la altura en la punta de los dedos.

En lo relativo a la evaluación de AP en las vistas laterales izquierda y derecha (Tabla II), la E0 muestra que la lordosis lumbar aumentada fue la más frecuente, seguida del aumento de la lordosis cervical y en igual número se observó alteración en la posición cefálica y la caída del hilo en los pies. Un hallazgo importante para señalar es la cifosis torácica que se mostró normal en todos los participantes.

En la E1 fue posible determinar modificaciones únicamente en las estructuras del componente corporal superior (cabeza y cuello) en ambas vistas laterales con la instalación del AOF. Posteriormente, en la E2 se estableció una influencia positiva sobre la posición cefálica del $50 \%$ de los participantes, alcanzando así el $100 \%$ en posición recta. La lordosis cervical incrementó igualmente el $50 \%$, para así alcanzar el $90 \%$ de la población con lordosis normal. En los demás parámetros evaluados también se observaron cabios positivos, a excepción de la cifosis torácica que no registró alteraciones desde la E0.

No se observaron diferencias entre las clases de Angle y los parámetros posturales evaluados en la E0 $\left(X^{2}=0,329 ; p>0,05\right)$, aunque en los pacientes con Clase I se observó menor número de parámetros en desequilibrio, esto no fue significativo. En relación al tipo de AOF utilizado, no se observaron diferencias significativas en la cantidad de parámetros afectados $\left(X^{2}=0,291 ; p>0,05\right)$. Se determinó que existió diferencia importante entre el número total de parámetros evaluados en los cuatro planos evaluados en E0 y E2 ( $p=0,007 ; p<0,05)$, con una reducción significativa de los mismos.

Se interrogó sobre la frecuencia de uso del AOF, la activación y la percepción que tuvieron de su funcionamiento; encontrando que solo un tutor mencionó inconsistencia con el uso del AOF, dos pacientes molestia y lesiones en tejidos blandos por presencia de márgenes agudos en los componentes del aparato, los cuales fueron resueltos en un tiempo menor a 1 semana. 


\section{DISCUSIÓN}

Existe una interacción dinámica constante entre las estructuras corporales, y el componente craneofacial refleja ésta conexión, existen estudios previos que soportan esta la teoría (Machado et al.; Silvestrini-Biavati et al.; Cossio-Escobar \& LemaÁlvarez et al., 2014).

Algunos autores como García (2008), Aguilar Moreno \& Taboada Aranza, han sugerido que la disfunción del sistema estomatognático afecta directamente la postura corporal. Las maloclusiones se presentan acompañadas de problemas posturales y ambos desórdenes son frecuentes en la población infantil que cruza por una etapa crucial debido a los cambios morfológicos y funcionales que se desarrollan, y además pueden influir en el establecimiento de alteraciones musculo esqueléticas.

Es por lo anterior se sugiere que la infancia es una etapa oportuna para realizar intervenciones terapéuticas que propicien una actitud corporal más idónea y una oclusión funcional.

Es una asociación tangible, tal como lo expresa Murrieta Pruneda, que la instalación de aparatos ortopédicos miofuncionales puede prevenir e interceptar problemas del sistema estomatognático para generar una armonía funcional que acompaña el desarrollo del cuerpo, de forma similar a lo observado en el presente estudio.

Estudios recientes, tal como el de Aguilar Moreno \& Taboada Aranza, muestran que es frecuente la presencia de maloclusión en personas con actitudes corporales incorrectas en poblaciones infantiles con maloclusión esquelética clase II, con un método de evaluación postural similar al utilizado para este estudio, interpretando como postura correcta o incorrecta, en lugar de equilibrado/desequilibrado, en los mismos planos y sistema de referencia. En dicho estudio, no se reporto un abordaje o seguimiento de la evaluación.

Respecto a la modificación de la postura ocasionada por el uso de dispositivos ortopédicos/ ortodónticos se ha reportado con anterioridad (Smailiene et al., 2017) que la terapéutica a través de Twin-block tras 10 a 14 meses de uso reduce significativamente la presencia de cifosis, lordosis, ángulos cervicales y torácicos y pelvis, de forma simi- lar a la observada en el presente estudio, sin embargo los cambios observados no son atribuidos por los investigadores a uso del dispositivo, si no al mero crecimiento corporal fisiológico. Al respecto nos es posible argumentar que la E1 refleja un efecto inmediato y la E2 la permanencia durante el periodo de uso, por lo que podemos asumir que los cambios observados son ocasionados por el AOF.

Una de las principales limitaciones del presente estudio es el tamaño de la población, solo un pequeño grupo cumplió con los criterios de selección preestablecidos, por lo tanto esto imposibilita la generalización de los resultados.

\section{CONCLUSIÓN}

La estrecha y continua interrelación entre las estructuras craneofaciales ejerce influencia sobre la postura, y su abordaje produce cambios evaluables desde distintas perspectivas, tal y como se muestra en éste estudio tras un periodo de dos meses de uso del AOF en el que se observaron cambios positivos en la actitud postural de todos los pacientes, lo que sugiere que la terapia miofuncional favorece al equilibrio estructural, aunque por limitaciones propias del estudio no se determinó la permanencia de las modificaciones.

ENRÍQUEZ, M. B.; DÍAZ, C. Y.; HERNÁNDEZ, M. E.; LUENGO, J. A. \& REYES, H. Determination of postural attitudes and their evolution after functional orthopedic approach. Descriptive study. Int. J. Odontostomat., 12(1):121127, 2018.

ABSTRACT: The objective of the study was to determine postural attitudes following the use of Functional orthotics (AOF) in pediatric patients with malocclusion. Descriptive, exploratory, longitudinal study, carried out in the Clinic of the Pediatric Dentistry Specialty (CLIO) of the UAO/ UAZ, during the period from January to September 2015. Patients with 6-12 years old of both sexes were included, through a non-probabilistic sampling at convenience. The presence or absence of postural balance was determined through photographs in anterior, posterior and lateral views (right / left) in three moments: prior to the installation of the AOF, 10 min after and after 10 weeks of use. The type of malocclusion according to Angle, the AOF used, and the number of parameters in disequilibrium in E0 and E2 were considered for the evaluation. The data collected were stored in the SPSS V-21 program and analyzed with the chi square 
and Wilcoxon tests, considering a value of $p<0.05$ as significant. In the study 10 patients were evaluated (mean age of $7.2 \pm 0.99$ years) and presented entirely abnormal postural attitudes in the sagittal and frontal planes. It was possible to determine a reduction of 60 and $80 \%$ of imbalance in some of the parameters evaluated (shoulder symmetry and height at the tips of the fingers), and these changes were manifested in a subtle way since the installation of the AOF and increased in the E2. Myofunctional therapy on patients with Angle malocclusions I and II is accompanied by changes in the postural attitude that can be observed from the installation of the devices, and these changes remain during the period of use.

KEY WORDS: postural attitude, malocclusion, functional orthopedic appliances.

\section{REFERENCIAS BIBLIOGRÁFICAS}

Aguilar Moreno, N. A. \& Taboada Aranza, O. Frecuencia de maloclusiones y su asociación con problemas de postura corporal en una población escolar del Estado de México. Bol. Med. Hosp. Infant. Mex., 70(5):364-71, 2013.

Baldini, A.; Nota, A.; Tripodi, D.; Longoni, S. \& Cozza, P. Evaluation of the correlation between dental occlusion and posture using a force platform. Clinics (Sao Paulo), 68(1):45-9, 2013.

Cavallé, M.; Brami, P.; De la Ballina, A.; Martín, M.; Peleato, M. \& Morcillo, F. Kinesiología Médica Odontológica y Posturología. Oviedo, Edikine, 2012.

Chessa, G.; Marino, A.; Dolci, A. \& Lai, V. Baropodometric examination for complete diagnosis of patients with craniocervico-mandibular disorders. Minerva Stomatol., 50(7-8):2718, 2001.

Cossio-Escobar, M. \& Lema-Álvarez, M. C. How our body asks for orthodontics. Rev. CES Odontol., 27(1):91-103, 2014.

Garcia de Paula e Silva, F. W.; Mussolino de Queiroz, A. \& DíazSerrano, K. V. Alteraciones posturales y su repercusión en el sistema estomatognático. Acta Odontol. Venez., 46(4), 2008. Disponible en: https://www.actaodontologica.com/ediciones/ 2008/4/art-22/

Kendall, F. \& McCreary, E. Músculos: Provas e Funções. São Paulo, Manoles Ltda., 1995.

Knutson, G. A. \& Jacob, M. Possible manifestation of temporomandibular joint dysfunction on chiropractic cervical $X$ ray studies. J. Manipulative Physiol. Ther., 22(1):32-7, 1999.

Machado, H.; Quirós, O.; Maza, P.; Fuenmayor, D.; Jurisic, A.; Alcedo, C. \& Ortiz, M. Correlación de la huella plantar y las maloclusiones en niños de 5 a 10 años que asisten a la Escuela Arturo Uslar Pietri en Maturín, Edo. Monagas. Rev. Latinoam. Ortod. Odontopediatria, (11), 2009. Disponible en: https:// www.ortodoncia.ws/publicaciones/2009/art11.asp

Martínez Medina, I. A.; Blero, A.; Navarro, C. S.; Ratia Martínez, F. \& Sánchez Aguilera, F. El dolor de espalda causado por malposiciones dentaria (Quinesiología dental, posturología y Odontología del deporte). Gac. Dent., (135):68-88. 2003.

Miralles, R.; Moya, H.; Ravera, M. J.; Santander, H.; Zúñiga, C.; Carvajal R. \& Yazigi, C. Increase of the vertical occlusal dimension by means of a removable orthodontic appliance and its effect on craniocervical relationships and position of the cervical spine in children. Cranio, 15(3):221-8, 1997.
Montero Parrilla, J. M. \& Denis Alfonso, J. A. Los trastornos temporomandibulares y la oclusión dentaria a la luz de la posturología moderna. Rev. Cuba. Estomatol., 50(4):408-21, 2013.

Murrieta Pruneda, J. F. Maloclusión dental y su relación con la postura corporal: un nuevo reto de investigación en Estomatología. Bol. Med. Hosp. Infant. Mex., 70(5):341-3, 2013.

Novo, M. J.; Changir, M. \& Quirós, A. O. Relación de las alteraciones plantares y las maloclusiones dentarias en niños. Rev. Latinoam. Ortod. Odontopediatria, (32), 2013. Disponible en: http://www.ortodoncia.ws/publicaciones/2013/art32.asp

Pereira Rosa, L.; de Moraes, L. C.; Leonelli de Moraes, M. E.; Medici Filho, E. \& de Melo Castilho, J. C. Evaluation of body posture associated with Class II and Class III malocclusion. Rev. Odonto. Ciênc., 23(1):20-5, 2008.

Silvestrini-Biavati, A.; Migliorati, M.; Demarziani, E.; Tecco, S.; Silvestrini-Biavati, P.; Polimeni, A. \& Saccucci, M. Clinical association between teeth malocclusions, wrong posture and ocular convergence disorders: an epidemiological investigation on primary school children. BMC Pediatr.,13:12, 2013.

Smailiene, D.; Intiene, A.; Dobradziejute, I. \& Kusleika, G. Effect of treatment with twin-block appliances on body posture in class II malocclusion subjects: A prospective clinical study. Med. Sci. Monit., 23:343-52, 2017

Dirección para correspondencia:

Cristal Yurixie Díaz Rosas

Programa de Especialidad en Odontopediatría

Unidad Académica de Odontología

Universidad Autónoma de Zacatecas (UAZ)

Carretera a la Bufa S/N

Colonia Centro Zacatecas

C.P. 98000

Zacatecas

MÉXICO

Email: yurixie_d@hotmail.com

Recibido : 30-11-2017

Aceptado: 06-01-2018 